\title{
Two-body bound and edge states in the extended SSH Bose-Hubbard model
}

\author{
M. Di Liberto ${ }^{1, a}$, A. Recati ${ }^{1,2}$, I. Carusotto $^{1}$, and C. Menotti ${ }^{1}$ \\ 1 INO-CNR BEC Center and Dipartimento di Fisica, Università di Trento, 38123 Povo, \\ Italy \\ 2 Arnold Sommerfeld Center for Theoretical Physics, Ludwig-Maximilians-Universität München, \\ 80333 München, Germany
}

\begin{abstract}
We study the bosonic two-body problem in a Su-SchriefferHeeger dimerized chain with on-site and nearest-neighbor interactions. We find two classes of bound states. The first, similar to the one induced by on-site interactions, has its center of mass on the strong link, whereas the second, existing only thanks to nearest-neighbors interactions, is centered on the weak link. We identify energy crossings between these states and analyse them using exact diagonalization and perturbation theory. In the presence of open boundary conditions, novel stronglylocalized edge-bound states appear in the spectrum as a consequence of the interplay between lattice geometry, on-site and nearest-neighbor interactions. Contrary to the case of purely on-site interactions, such EBS persist even in the strongly interacting regime.
\end{abstract}

\section{Introduction}

A peculiar feature of interacting lattice models is the existence of two-particle bound states for both attractive and repulsive interactions. This can be intuitively understood as a consequence of the bounded kinetic energy bandwidth, which does not allow dissipation of large interaction energies [1-7]. For repulsive interactions, such doublons are therefore stable high-energy composite objects with their own dynamics and properties. In the presence of nearest-neighbor interactions, the picture becomes even richer because stable objects can be formed by two particles sitting on neighboring sites $[4,5,8,9]$.

In recent years, the study of topological states of matter has disclosed a novel class of fascinating quantum phases, now well understood and classified at the singleparticle level [10]. A key feature of these states is the presence of edge modes that are robust and protected by symmetries. Much less understood is the role of interactions on these phases. On one hand, interactions can be responsible of very non-trivial many-body topological states, as the notorious fractional Quantum Hall effect [11]. On the other hand, interactions can have a detrimental effect on the single-particle topological phases [12].

In Ref. [13], we have shown that non-trivial physics arises for two interacting particles in the simplest topological lattice model, namely the Su-Schrieffer-Heeger

\footnotetext{
a e-mail: marco.diliberto@unitn.it
} 
chain, despite the fact that two-body on-site interactions reduce the symmetries protecting the single-particle edge modes. Indeed, for moderate values of on-site interactions, two-body edge modes may be found, the origin of which is attributed to the interplay between lattice geometry, topology and interactions themselves (see also [14]). These states can be understood as interaction-induced Tamm-Schockley surface modes $[15,16]$.

In this work, we extend our previous analysis including also nearest-neighbor interactions. We identify a new class of two-body out-of-cell bound states and study the resonances appearing in the spectrum when the energy of the new dimers matches the energy of the in-cell bound states studied in [13]. For finite chains, the presence of two-body edge bound states (EBS) is revealed in both dimerizations, with novel and enhanced localization properties due to nearest-neighbor interactions. We analyze the properties of bound and edge-bound states using exact diagonalization and effective theories in the strong-dimerization or strong-interaction limit.

Upon a mapping of the two-body wave function in one dimension (1D) onto a free particle wave function in an appropriately engineered two-dimensional (2D) lattice $[13,17,18]$, on-site interactions in 1D are translated onto an energy off-set in the main diagonal of the 2D lattice. Nearest-neighbor interactions can be analogously translated onto energy off-sets in the adjacent diagonals. In optical fiber setups, this mapping can be implemented through refractive-index modulations. At the two-body level, this makes it possible to realize nearest-neighbor interactions of arbitrary intensity, much harder to obtain in experiments with ultracold atoms [22]. Hence, optical fiber setups are probably the best candidates to explore experimentally our findings [19-21].

The paper is organized as follows. In Sec. 2, we introduce our model describing interacting particles in a dimerized lattice of alternating strong and weak links. In Sec. 3, we describe two classes of dimer states appearing when the strong links are decoupled from each other. We relate the first class to the in-cell dimer states found in [13] and discuss the completely different nature of the second class of out-of-cell dimers induced by nearest-neighbor interactions. In Sec. 4, we introduce a finite weak tunneling to allow dimer mobility and create delocalized two-body bound states. In particular, we discuss the resonances occurring between the two classes of bound states. In Sec. 5, we focus on the case of open boundary conditions and analyze the effect of nearest-neighbor interactions on the two-body bound edge states. In Sec. 6, we draw our conclusions.

\section{Model Hamiltonian}

The Su-Shrieffer-Heeger model ( $\mathrm{SSH}$ ) describes a single particle moving on a onedimensional lattice where the nearest-neighbor hopping coefficients alternate in magnitude. The single-particle SSH Hamiltonian reads [23]

$$
H_{0}=-J_{1} \sum_{i} c_{A, i}^{\dagger} c_{B, i}-J_{2} \sum_{i} c_{A, i+1}^{\dagger} c_{B, i}+\text { H.c. . }
$$

We consider bosonic particles interacting with on-site interactions

$$
H_{U}=\frac{U}{2} \sum_{i}\left[n_{A, i}\left(n_{A, i}-1\right)+n_{B, i}\left(n_{B, i}-1\right)\right]
$$

and nearest-neighbor interactions

$$
H_{V}=V \sum_{i}\left(n_{A, i} n_{B, i}+n_{A, i+1} n_{B, i}\right)
$$




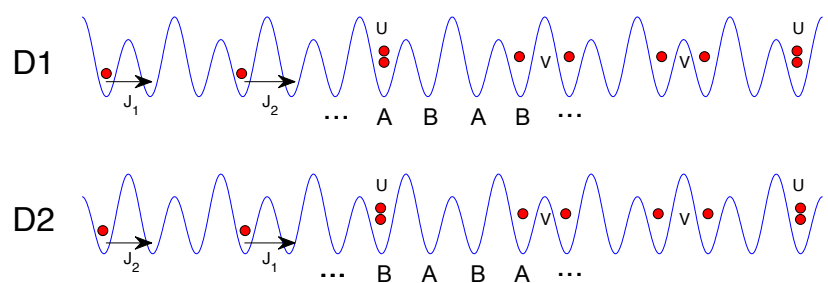

Fig. 1. Sketch of the SSH model with on-site and nearest-neighbor interactions. For open boundary conditions (OBC) and even number of lattice cells, one can have two type of dimerizations D1 and D2 depending on whether the chain ends with strong or weak links.

where $n_{\sigma, i} \equiv c_{\sigma, i}^{\dagger} c_{\sigma, i}$ is the density operator, $\sigma=A, B$ indicates the lattice site and $i$ labels the lattice cell (see Fig. 1).

In this work, we focus on the two-body physics in the strongly-dimerized regime, namely $J_{2} \ll J_{1}$. This naturally allows us to define as strong link those pairs of lattice sites $A_{i}$ and $B_{i}$ belonging to the same lattice cell $i$, and as weak link those pairs of neighboring lattice sites $B_{i}$ and $A_{i+1}$ belonging to two neighboring lattice cells. The interplay between in-cell kinetic energy $H_{J_{1}}=-J_{1} \sum_{i} c_{A, i}^{\dagger} c_{B, i}+$ H.c. and interactions determines the two-body states in the fully dimerized case $\left(J_{2}=0\right)$. The effect of the weak tunneling Hamiltonian between different cells $H_{J_{2}}=-J_{2} \sum_{i} c_{A, i+1}^{\dagger} c_{B, i}+$ H.c. will be considered exactly in the numerical simulations and introduced analytically at the perturbative level. For open boundary conditions (OBC) and even number of cells, two dimerizations are possible, as sketched in Fig. 1(D1,D2).

\section{Dimer states}

First, we consider the fully dimerized case of $J_{2}=0$. Bound pairs can be formed in the same lattice cell, as in the case of on-site interactions [13,24], or in neighboring lattice cells, exclusively induced by nearest-neighbor interactions.

As previously introduced in [13], the Hilbert space of the strong-link in-cell Hamiltonian is spanned by the states $\left|A_{i} A_{i}\right\rangle,\left|A_{i} B_{i}\right\rangle$ and $\left|B_{i} B_{i}\right\rangle$. In the presence of on-site and nearest-neighbors interactions, the Hamiltonian reads

$$
H_{i}^{\text {cell }}=\left(\begin{array}{ccc}
U & -\sqrt{2} J_{1} & 0 \\
-\sqrt{2} J_{1} & V & -\sqrt{2} J_{1} \\
0 & -\sqrt{2} J_{1} & U
\end{array}\right) .
$$

This Hamiltonian determines three dimer states $d_{\alpha}$, respectively at energies $\epsilon_{1,3}=$ $\frac{1}{2}\left(U+V \mp \sqrt{16 J_{1}^{2}+(U-V)^{2}}\right)$ and $\epsilon_{2}=U$. The nature of these states is similar to the one discussed in [13] for $V=0$. In fact, as far as the in-cell dimer wave functions are concerned, $V$ induces nothing else than a renormalized on-site interaction $U-V$. Hence, a repulsive nearest-neighbor interaction $V$ favours on-site occupation in $d_{1}$ and off-site occupation in $d_{3}$, leaving $d_{2}$ unaltered.

Dimer states of completely different nature are induced by the presence of nearestneighbor interaction between two atoms in neighboring cells. The Hilbert space properly taking into account out-of-cell dimers is spanned by the states $\left[\left|A_{i} A_{i+1}\right\rangle+\right.$ $\left.\left|B_{i} B_{i+1}\right\rangle\right] / \sqrt{2},\left|A_{i} B_{i+1}\right\rangle$ and $\left|B_{i} A_{i+1}\right\rangle$. We do not include in this subspace the fourth 
out-of-cell state $\left[\left|A_{i} A_{i+1}\right\rangle-\left|B_{i} B_{i+1}\right\rangle\right] / \sqrt{2}$ at zero energy, which is decoupled by the rest. In the considered subspace, the Hamiltonian reads

$$
H_{i+1 / 2}^{\text {nn-cells }}=\left(\begin{array}{ccc}
0 & -\sqrt{2} J_{1} & -\sqrt{2} J_{1} \\
-\sqrt{2} J_{1} & 0 & 0 \\
-\sqrt{2} J_{1} & 0 & V
\end{array}\right),
$$

where the subscript $i+1 / 2$ indicates the center-of-mass of the out-of-cell dimers. The out-of-cell Hamiltonian predicts three dimer states $\left|\tilde{d}_{\beta}\right\rangle^{1}$. Their energies $\tilde{\epsilon}_{\beta}$ cannot be computed analytically, but two important limits can be identified: (i) for $V \rightarrow 0$, one finds $\tilde{\epsilon}_{\beta}=\left[-2 J_{1}, 0,2 J_{1}\right]$ and the corresponding eigenstates tend to states of the three (type I) scattering continua around energy $\tilde{\epsilon}_{\beta}$; (ii) for $V \rightarrow \infty$, one finds $\tilde{\epsilon}_{\beta}=\left[-\sqrt{2} J_{1}, \sqrt{2} J_{1}, V\right]$ corresponding to the eigenstates $[1 ;-1 ; 0],[1 ; 1 ; 0]$, and $[0 ; 0 ; 1]$ written in our basis. The latter, $\left|B_{i}, A_{i+1}\right\rangle$, is obviously the state which maximizes nearest-neighbor interaction energy and is the off-site nearest-neighbor analog of the two in-cell states $d_{2}$ and $d_{3}$ in the large $U$ limit. The two states at $\pm \sqrt{2} J_{1}$ are instead the out-of-cell analog of state $d_{1}$ at $V=0$, minimizing the interaction energy. The effect of nearest-neighbor interactions on such states of two delocalized particles in neighboring cells is to enhance the occupation of one particle in the outer most site of one cell, leaving the second particle delocalized in the other cell. Due to the suppression of nearest-neighbor occupation, the kinetic energy is reduced to the asymptotic value of $\tilde{\epsilon}_{1,2}= \pm \sqrt{2} J_{1}$

It is useful to notice that at $U=0$, the six dimer states always appear in the sequence $\left[\tilde{\epsilon}_{1}, \epsilon_{1}, \epsilon_{2}, \tilde{\epsilon}_{2}, \tilde{\epsilon}_{3}, \epsilon_{3}\right]$. Knowing the asymptotic values of the energies as a function of $U$ and $V$, one can predict the presence of energy level crossings.

\section{Bound states}

In the presence of a small but finite hopping $J_{2}$, the dimers are allowed to move in the lattice developing narrow bound-state bands. Consequently, the full two-body energy spectrum - obtained e.g. in Fig. 2 by exact diagonalization with periodic boundary conditions (PBC) - shows three (type I) scattering continua, typical of the non-interacting two-body SSH model, and six bound-state bands. For dominant $U$ and small $V$, the out-of-cell bound states may be well defined only for certain values of the center-of-mass momentum $K$ either in the Brillouin zone center or boundary (see Fig. 2(a)). The in-cell and out-of-cell character of the different bound states is evident in their wave function plotted in Fig. 3. The bound states $d_{\alpha}$ and $\tilde{d}_{\beta}$ for $U=2.5$ and $V=3$ are respectively shown in Figs. 3(a-c) and Figs. 3(d-f).

To provide some more understanding of the general behaviour of the system, we plot the spectrum at fixed $U$ and varying $V$ in Fig. 4(a) and the spectrum at fixed $V$ and varying $U$ in Fig. 4(b). The behaviour of the bare dimer energies $\epsilon_{\alpha}$ and $\tilde{\epsilon}_{\beta}$ can be easily recognized in those figures. In Fig. 4, one can observe several crossings occurring. At those points, one of the out-of-cell bound states $\left|\tilde{d}_{\beta}\right\rangle$ becomes resonant with one of the in-cell bound states $\left|d_{\alpha}\right\rangle$. Similarly to what happens in the uniform Bose-Hubbard model in the presence of on-site and nearest-neighbors interactions, at the resonance conditions doublons acquire a much larger bandwidth. In fact, in contrast to the standard case where doublons move via second order processes at order

\footnotetext{
${ }^{1}$ Out-of-cell dimer states are reminiscent of the out-of-cell $d_{N N}$ state discussed in [13], which was formed by an effective nearest-neighbor interaction due to second-order coupling between different dimer states (see [13], Appendix 3). In the presence of a small but finite nearest-neighbor interaction $V$, state $d_{N N}$ is replaced by $\tilde{d}_{3}$.
} 
(a)

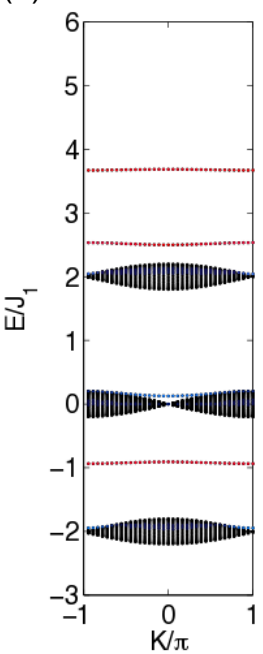

(b)

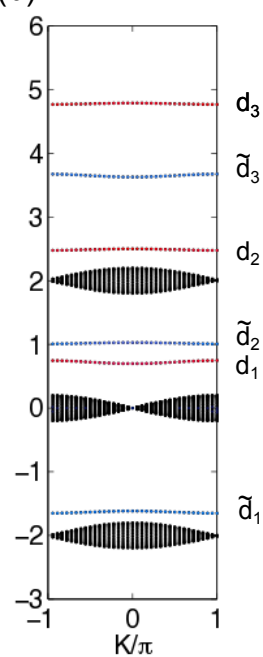

(c)

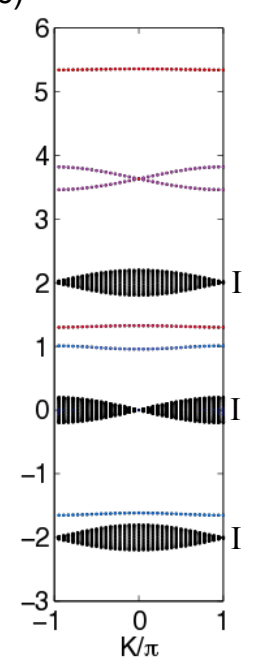

Fig. 2. Two-body energy spectrum for PBC as a function of the center of mass momentum $K$ for 72 lattice sites (36 lattice cells) for $J_{2}=0.1 J_{1}, U=3.63 J_{1}$ and different values of $V$ : (a) $V=0.3 J_{1}$; (b) $V=2 J_{1}$; (c) $V=3 J_{1}$. Red colorscale indicates relative in-cell population, while blue colorscale indicates relative nearest-neighbor out-of-cell population.

(a)

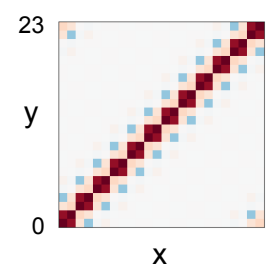

(d)

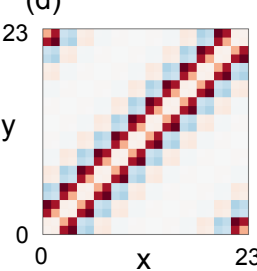

(b)

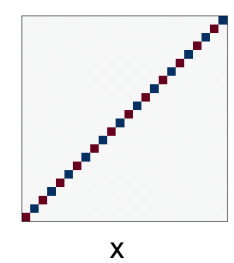

(e)

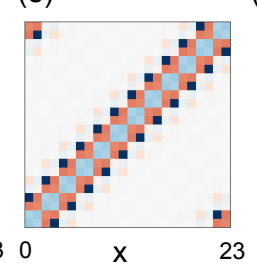

(c)

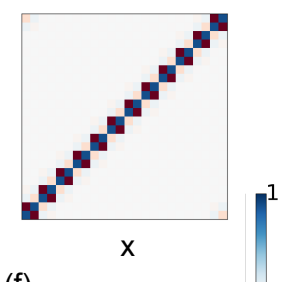

(f)

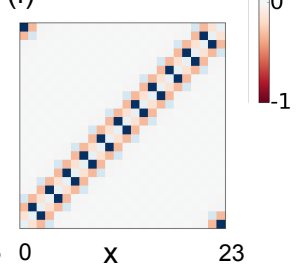

Fig. 3. (a-c) Bound state wavefunction $\left|d_{1}\right\rangle,\left|d_{2}\right\rangle,\left|d_{3}\right\rangle$ at $K=0$ for $J_{1}=0.1 J_{1}, U=2.5 J_{1}$, and $V=3 J_{1}$; (d-f) Bound state wavefunctions $\left|\tilde{d}_{1}\right\rangle,\left|\tilde{d}_{2}\right\rangle,\left|\tilde{d}_{3}\right\rangle$ at $K=0$ for $J_{2}=0.1 J_{2}$, $U=2.5 J_{1}$ and $V=3 J_{1}$. The color code is normalized to the maximum absolute value of the wave function.

$J_{2}^{2}$, in-cell bound states and out-of-cell bound states are now resonantly coupled at first order in $J_{2}$. Each single-particle hopping induces the motion of the center-of-mass of the doublon of half a lattice cell. This effective reduction of the lattice spacing is translated into an effective doubling of the Brillouin zone, as observed in Fig. 2(c), where the two bound-state bands relative to $\left|d_{\alpha}\right\rangle$ and $\left|\tilde{d}_{\beta}\right\rangle$ merge into a single one (in the specific case $\alpha=2$ and $\beta=3$ ). Correspondingly, the wave functions are completely hybridized, showing similar in-cell and out-of-cell populations (see Fig. 5(a-c)). 
(a)

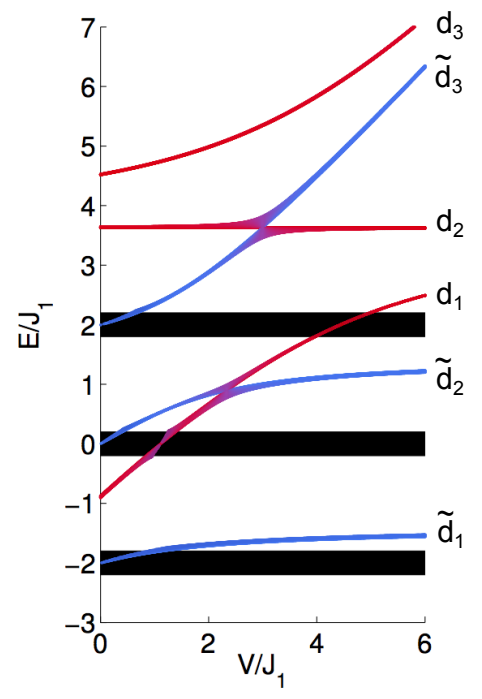

(b)

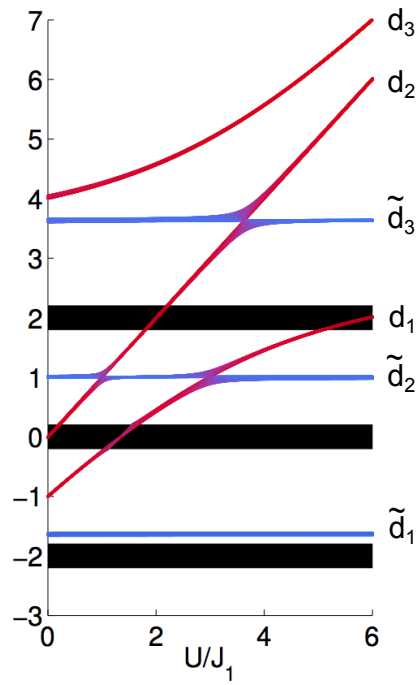

Fig. 4. (a) Spectrum for $J_{2}=0.1 J_{1}, U=3.63 J_{1}$ as a function of $V$ and (b) spectrum for $J_{2}=0.1 J_{1}, V=3 J_{1}$ as a function of $U$ obtained by exact diagonalization of a lattice of $L=48$ sites (24 cells) with PBC. Red colorscale indicates relative in-cell population, while blue colorscale indicates relative nearest-neighbor out-of-cell population.

(a)

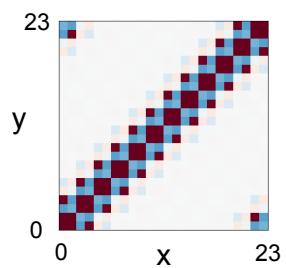

(b)

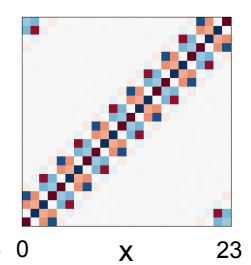

(c)

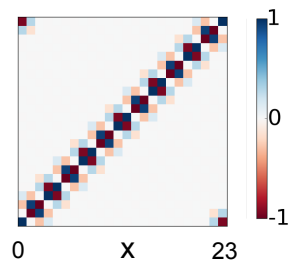

Fig. 5. Examples of hybridized bound-state wave functions for $V=3 J_{1}$ (see Fig. 4(b)) at the resonance points (a) $U=J_{1}$, (b) $U=3 J_{1}$ and (c) $U=3.63 J_{1}$. The color code is normalized to the maximum absolute value of the wave function.

Bound-state energy crossings can be described by an effective theory. For simplicity of notation, we call $|d\rangle$ and $|\tilde{d}\rangle$ the two in-cell and out-of-cell bound states involved in the crossing. Following the standard approach [25], as outlined in the appendix of [13], we define an effective Hamiltonian $H_{\text {eff }}$ that includes $H_{J_{2}}$ as a perturbation. Hopping between states $|d\rangle$ and $|\tilde{d}\rangle$ takes place at first order in $J_{2}$ and is accounted for by the effective hopping matrix elements

$$
J_{\text {eff }} \equiv\left\langle\tilde{d}_{i+1 / 2}\left|H_{\text {eff }}\right| d_{i}\right\rangle \approx\left\langle\tilde{d}_{i+1 / 2}\left|H_{J_{2}}\right| d_{i}\right\rangle .
$$

Analogously, one obtains $\left\langle\tilde{d}_{i-1 / 2}\left|H_{J_{2}}\right| d_{i}\right\rangle=-J_{\text {eff }}$. We recall that the subscript $i$ $(i+1 / 2)$ indicates the center-of-mass of the in-cell (out-of-cell) dimers. For periodic boundary conditions (PBC), the effective Hamiltonian parameters do not depend on $i$. The effective model thus reads

$$
H_{\mathrm{eff}}=\sum_{i}\left(\epsilon d_{i}^{\dagger} d_{i}+\tilde{\epsilon} \tilde{d}_{i+1 / 2}^{\dagger} \tilde{d}_{i+1 / 2}\right)+J_{\mathrm{eff}} \sum_{i}\left(d_{i}^{\dagger} \tilde{d}_{i+1 / 2}-d_{i}^{\dagger} \tilde{d}_{i-1 / 2}+\text { H.c. }\right) \text {. }
$$


Such effective model describes a single-particle in a superlattice with alternating potential energy offsets $\epsilon$ and $\tilde{\epsilon}$. When the resonance condition $\epsilon=\tilde{\epsilon}$ is met, a uniform lattice is obtained, recovering the case of Fig. 2(c). The alternating sign of the hopping terms is irrelevant and can be reabsorbed through a gauge transformation. tions

For the specific case of $|d\rangle=\left|d_{2}\right\rangle$ and $|\tilde{d}\rangle=\left|\tilde{d}_{3}\right\rangle$, one can use the dimer wavefunc-

$$
\begin{aligned}
\left|d_{2, i}\right\rangle & =\frac{1}{\sqrt{2}}\left(\left|A_{i} A_{i}\right\rangle-\left|B_{i} B_{i}\right\rangle\right), \\
\left|\tilde{d}_{3, i+1 / 2}\right\rangle & =\frac{D_{1}}{\sqrt{2}}\left(\left|A_{i} A_{i+1}\right\rangle+\left|B_{i} B_{i+1}\right\rangle\right)+D_{2}\left|A_{i} B_{i+1}\right\rangle+D_{3}\left|B_{i} A_{i+1}\right\rangle,
\end{aligned}
$$

where the coefficients $D_{n}$ depend on $U$ and $V$ and are obtained from the diagonalization of Eq. (5). Hence, the Hamiltonian parameters read

$$
\epsilon=\epsilon_{2}, \quad \tilde{\epsilon}=\tilde{\epsilon}_{3}, \quad J_{\mathrm{eff}}=D_{3} J_{2}
$$

This effective theory reproduces exactly the bound state crossing occurring for $V=$ $3 J_{1}$ around $U \sim 3.63 J_{1}$.

\section{Edge bound states}

Let us now consider the case of open boundary conditions (OBC) and focus our attention on the presence of edge bound states (EBS), defined as a bound pair localized at the edges of the chain, hence displaying localization both in the relative and center of mass coordinates.

In dimerizations $D 1$ (see Fig. $1(\mathrm{D} 1)$ ), the existence of the $D 1 / d_{3}$ EBS for small $U$ is confirmed also when nearest-neighbor interactions are present. The most noteworthy feature introduced by $V$ is a novel strongly localized EBS related to bound state $d_{1}$ (see lower green line in Fig. 6(a)). This state, absent for $V=0$, exists for generic $U \gtrsim V>0$. Notice that there is an interesting correspondence between $D 1 / d_{3}$ EBS at small $U$ and $D 1 / d_{1}$ EBS at large $U$. In fact, in both cases the wavefunction is related to dimer state $\left|A_{i} B_{i}\right\rangle$, which possesses large energy $V$ for $0<U \ll V$ and small energy $V$ for $J_{1} \ll V \ll U$. For increasing $U$ or $V$ respectively, admixture with dimer states $\left|A_{i} A_{i}\right\rangle$ and $\left|B_{i} B_{i}\right\rangle$ occurs and the EBS eventually lose localization. The origin of $D 1 / d_{1}$ and $D 1 / d_{3}$ EBS will be explained based on a Tamm-like model at the end of this section.

In dimerization $D 2$ (see Fig. 1(D2)), as clearly visible by the green lines in Fig. 6(b), very pronounced EBS appear. At small $V$, these EBS arise at energy close to $\pm J_{1}$ and are reminiscent of the $D 2 / d_{1,2}$ EBS discussed in [13], namely on-site edge doublons undergoing a mixing with a single-particle edge state plus a free scattering particle (denoted as type II scattering continuum). These EBS can be predicted by the same reduced theory developed in [13] (see matrix $\mathcal{H}^{\text {red }}$ in Eq. (A25)), upon introducing the appropriate corrections due to nearest-neighbor interactions. Apart from on-site edge doublons and type II scattering states, the basis of the reduced theory has now to contain out-of-cell edge doublons. Hence, the starting point of the reduced theory is a proper truncation ansatz for the states at the edges. For instance, in the specific case of states $\left|d_{2}\right\rangle$ and $\left|\tilde{d}_{3}\right\rangle$, at the left edge of the lattice one can define

$$
\left|d_{2,0}\right\rangle=-\left|B_{0} B_{0}\right\rangle, \quad\left|\tilde{d}_{3,1 / 2}\right\rangle=D_{3}^{L}\left|B_{0} A_{1}\right\rangle+D_{1}^{L}\left|B_{0} B_{1}\right\rangle .
$$


(a)

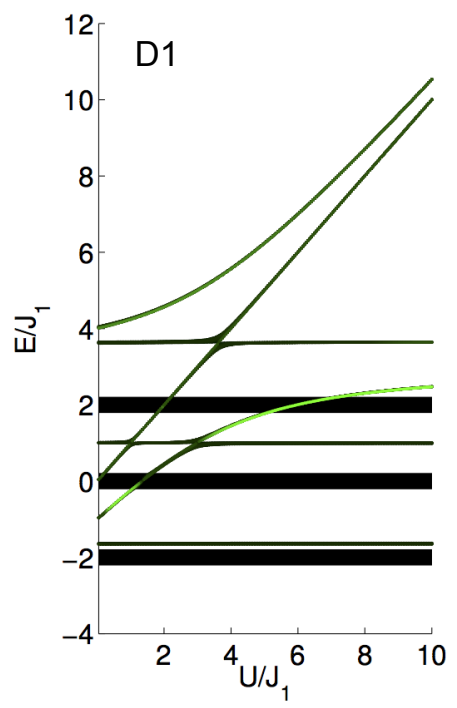

(b)

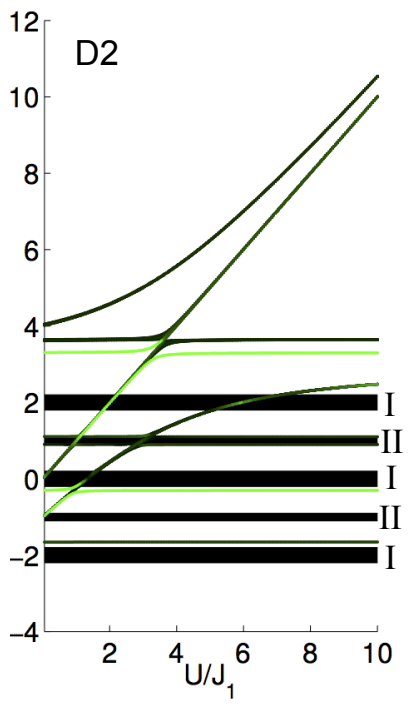

Fig. 6. Spectrum as a function of $U$ for $J_{2}=0.1 J_{1}$ and nearest-neighbor interaction $V=3 J_{1}$ in (a) dimerization $D 1$ and (b) dimerization $D 2$, obtained by exact diagonalization of a lattice with OBC for $L=48$ sites ( 24 cells). Green colorscale indicates the relative population in the first 4 lattices sites (2 lattice cells) which highlights the edge localization of the states.

While state $\left|d_{2,0}\right\rangle$ is the same one considered in [13], state $\left|\tilde{d}_{3,1 / 2}\right\rangle$ differs from $\left|B_{0}\right\rangle \otimes$ $\left(\left|A_{1}\right\rangle-\left|B_{1}\right\rangle\right)$ by a renormalization of the coefficients due to $V$. More precisely, $D_{1}^{L}$ and $D_{3}^{L}$ depend on $V$ and are obtained from the eigenvector of the matrix

$$
H_{1 / 2}^{\mathrm{nn}-\mathrm{cells}}=\left(\begin{array}{cc}
V & -J_{1} \\
-J_{1} & 0
\end{array}\right)
$$

corresponding to the largest eigenvalue $\lambda_{+}=\left(V+\sqrt{V^{2}+4 J_{1}^{2}}\right) / 2$. Consequently, the matrix $\mathcal{H}^{\text {red }}$ is identical to the one in Eq. (A25) of Ref. [13], apart for the elements involving $\left|\tilde{d}_{3,1 / 2}\right\rangle$. The upper left corner of $\mathcal{H}^{\text {red }}$, which describes the left edge physics, becomes

$$
\mathcal{H}_{L}^{\mathrm{red}}=\left(\begin{array}{ccc}
U & -\sqrt{2} J_{2} D_{3}^{L} & 0 \\
-\sqrt{2} J_{2} D_{3}^{L} & \lambda_{+} & -J_{2} D_{1}^{L} / \sqrt{2} \\
0 & -J_{2} D_{1}^{L} / \sqrt{2} & J_{1}
\end{array}\right) .
$$

An analogous treatment holds for the right edge, modifying the lower right corner of $\mathcal{H}^{\text {red }}$. This modified theory predicts the existence of two possible kinds of EBS, namely $D 2 / d_{2}$ and the novel $D 2 / \tilde{d}_{3}$ (upper green diagonal and horizontal lines in Fig. 6(b), respectively). The new feature, due to nearest-neighbor interactions, is the presence of the $D 2 / \tilde{d}_{3}$ EBS. For $V=0$, the $D 2 / d_{2}$ EBS smoothly transforms into a type II scattering state as $U$ moves away from $J_{1}$ (see Ref. [13], Fig. 7). Instead when scanning $U$ in the presence of $V \neq 0$, the $D 2 / d_{2}$ EBS smoothly becomes a $D 2 / \tilde{d}_{3}$ EBS. Near the avoided crossing, the two EBS hybridize. The $[2(L-1)+3] \times[2(L-1)+3]$ matrix $\mathcal{H}^{\text {red }}$ (where $L$ is the number of cells in the lattice) correctly accounts for the energy shift of the $D 2 / \tilde{d}_{3}$ EBS above type II scattering continuum even for very small values of $V$. The effect of $V$ is to bring the two-body edge components of the 
(a)

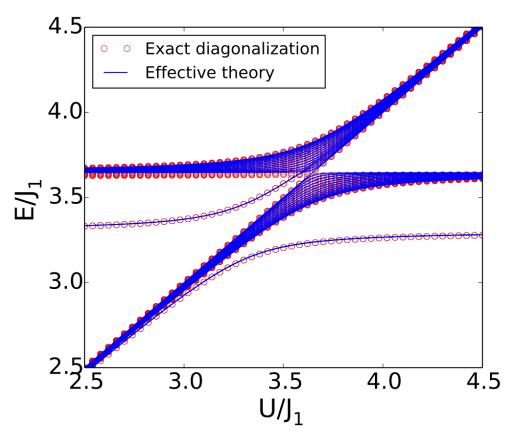

(b)

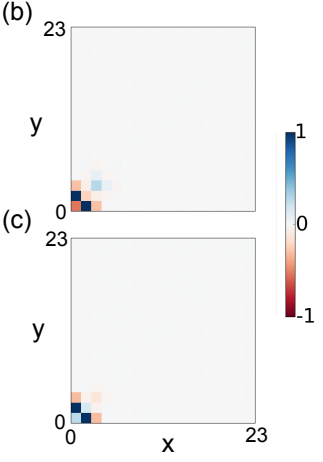

Fig. 7. (a) Closer view of the spectrum with $\mathrm{OBC}$ in dimerization $D 2$ with the same parameters as in Fig. 6(b) for 36 lattice sites. A comparison between the exact spectrum and the one obtained from the effective theory in (7),(13) is shown. (b-c) Edge bound states for $U=3 J_{1}$ (b) and $U=4 J_{1}$ (c). The color code is normalized to the maximum absolute value of the wave function.

edge/free particle states out of resonance with the rest of type II scattering continuum. Eventually, for sufficiently large $V, D_{1}^{L} \rightarrow 0$, such that $\left|d_{2,0}\right\rangle$ and $\left|\tilde{d}_{3,0}\right\rangle$ decouple from the type II continuum. One can therefore simplify the description provided by the complete $\mathcal{H}^{\text {red }}$ matrix by taking only the first $2 \times 2$ block of $\mathcal{H}_{L}^{\text {red }}$ in Eq. (12) for the left edge, and an analogous $2 \times 2$ block for the right edge.

The accuracy of the $2 \times 2$ model proves excellent in reproducing the avoided crossing in Fig. 6(b) (upper green curves). For $V \sim U$, namely when nearest-neighbor interactions and on-site interactions are of the same order, the presence of the $d_{2}$ and $\tilde{d}_{3}$ bound state narrow bands becomes relevant. The $D 2 / d_{2}$ EBS predicted with the reduced theory has no numerical evidence for sufficiently large $U$, such that $\epsilon_{2} \gtrsim \tilde{\epsilon}_{3}$. To include the effect of the $d_{2}$ and $\tilde{d}_{3}$ bound state narrow bands, we can resort to the effective model presented in Eq. (7). Considering the modified states at the edges as in Eq. (10), one finds

$$
\epsilon^{\text {edge }}=\epsilon=U, \quad \tilde{\epsilon}^{\text {edge }}=\frac{V+\sqrt{V^{2}+4 J_{1}^{2}}}{2} \neq \tilde{\epsilon}, \quad J_{\text {eff }}^{\text {edge }}=\sqrt{2} D_{3}^{L} J_{2},
$$

providing a substantial renormalization of the edge parameters with respect to the bulk $^{2}$. In this respect, the emerging EBS can be interpreted as Tamm-like states. When $U$ is sufficiently far from the value where the crossing takes place and hybridization with other states does not occur, the $D 2 / \tilde{d}_{3}$ EBS arises mainly thanks to $\tilde{\epsilon}^{\text {edge }}$ and its energy becomes independent of $U$. As shown in Fig. 7(a), around the level crossing the agreement between effective model and numerical results is excellent. Since higher type II scattering continuum and bound state $d_{3}$ are not included in model (7),(13), the conditions required for an accurate prediction of the bound state bandwidth and the EBS energies are $U \sim V$ and $V>2 J_{2}$, respectively. At fixed $U$ and for large $V$, the $D 2 / \tilde{d}_{3}$ EBS persists, preserving excellent localization properties. This is due to the fact that, even if its energy gap with respect to the $\left|\tilde{d}_{3}\right\rangle$ bound-state

\footnotetext{
2 Since the basis states (10) are in common between the effective models (12) and (7),(13), it is not a coincidence that the renormalized parameters in (13) coincide with the matrix elements of (12).
} 
(a)

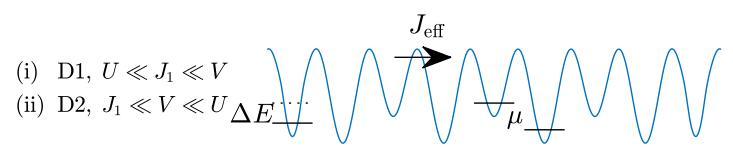

(iii) $\mathrm{D} 1, J_{1} \ll V \ll U$

(iv) $\mathrm{D} 2, U \ll J_{1} \ll V$

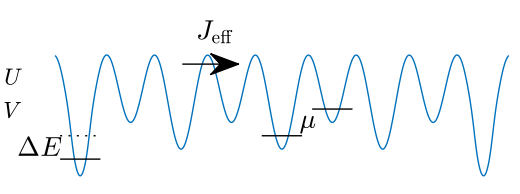

(b)

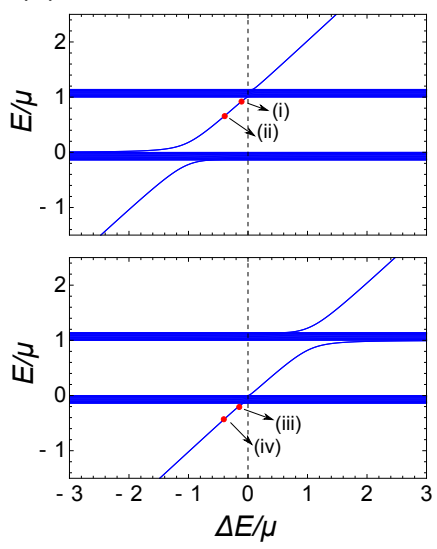

Fig. 8. (a) Effective single-particle ionic Hubbard model with hopping $J_{\text {eff }}$, potential energy off-set $\mu$, and edge potential energy correction $\Delta E$; The boundary conditions in the upper panels accounts for the cases (i)-(ii), while the boundary conditions in the lower panels account for the cases (iii)-(iv), as indicated. (b) Tamm analysis for the effective ionic Hubbard model for fixed $\mu$ and $J_{\text {eff }}$ and varying $\Delta E$; The zero of the energy is set to the lowest bulk energy offset; The red dots indicate the prediction for the edge modes for the four cases: (i) $D 1 / d_{3}$ EBS just below the upper $\left(d_{3}\right)$ continuum for $U \ll J_{1} \ll V$; (ii) novel strongly localized $D 2 / \tilde{d}_{3}$ EBS in between the lower $\left(d_{1}\right)$ and the upper $\left(\tilde{d}_{3}\right)$ continua with gap $\mu / 2$ for $J_{1} \ll V \ll U$; (iii) novel $D 1 / d_{1}$ EBS just below the lower $\left(d_{1}\right)$ continuum for $J_{1} \ll V \ll U$; (iv) novel strongly localized $D 2 / \tilde{d}_{3}$ EBS below the lower $\left(\tilde{d}_{3}\right)$ continuum with gap $\mu / 2$ for $U \ll J_{1} \ll V$.

narrow band becomes smaller and smaller, the ratio between the gap and the $\left|\tilde{d}_{3}\right\rangle$ bandwidth remains finite, preventing EBS diffusion.

A unified picture to describe the novel $D 1 / d_{1}$ and $D 2 / \tilde{d}_{3}$ EBS can be obtained resorting to an effective model in the strongly interacting regime. For both $D 1$ and $D 2$, the states involved are $d_{3}$ and $\tilde{d}_{3}$ in the limit $U \ll J_{1} \ll V$ and $d_{1}$ and $\tilde{d}_{3}$ in the limit $J_{1} \ll V \ll U$. Dimers states $\left|d_{3}\right\rangle$ for $U \ll V$ and $\left|d_{1}\right\rangle$ for $U \gg V$ are accounted for by the same ansatz $\left|A_{i} B_{i}\right\rangle$, and dimer $\left|\tilde{d}_{3}\right\rangle$ is approximated by $\left|A_{i} B_{i+1}\right\rangle$. Contrary to the effective model presented in Sec. 4, both hopping terms $J_{1}$ and $J_{2}$ are now considered at the pertubative level, through second order processes. One obtains an effective single-particle ionic Hubbard model with constant hopping parameter $J_{\text {eff }}=J_{1} J_{2}(1 / V+2 /(V-U))$ and alternating potential energy offsets. The underlying effective lattice sites correspond to pairs of sites of the original lattice. The energy offsets in the effective lattice are given by the energy of the corresponding (either in-cell or out-of-cell) dimer states in the original lattice, leading to a site-tosite energy difference $\mu=2\left(J_{1}^{2}-J_{2}^{2}\right)|1 / V-2 /(V-U)|$. Depending on whether the energy offset at the boundary of the effective lattice is the largest or the smallest (which depends at once on the dimerization $D 1$ or $D 2$ of the original lattice and on the relation between $U$ and $V$ ), the four cases under consideration are represented by one or the other effective ionic Hubbard model sketched in Fig. 8(a) (see labels in figure for a better understanding). Moreover, depending on the dimerization $D 1$ or $D 2$ of the original lattice, open boundary conditions introduce at the outmost sites a further energy off-set $\Delta E$, as sketched in Fig. 8(a).

The spectrum of the ionic Hubbard model presents two continua respectively below and above energies 0 and $\mu$, with bandwidth $\mathcal{W}=\left|\mu-\sqrt{\mu^{2}+16 J_{\text {eff }}^{2}}\right| / 2$. Depending 
on $\Delta E$ and on the boundary conditions, the Tamm-analysis generalized to the ionic Hubbard model, shown in Fig. 8(b), predicts that the system can support gapped edge modes either below or above the continua, or in the gap (see Fig. 8(b)).

In dimerization $D 1$, both for $U \ll J_{1} \ll V$ and $J_{1} \ll V \ll U$, one obtains $\Delta E=-J_{2}^{2} / V$, which is much smaller than $\mu$ in the limit $J_{2} \ll J_{1}$. This energy correction, even if very small, allows for edge modes just below the upper continuum (i) and even below the lower continuum (iii). In case (i), the Tamm analysis reveals that any small $\Delta E<0$ is sufficient to localize states at the edges. In case (iii), the required condition is $|\Delta E|>\mathcal{W} / 2$. A perturbative calculation shows that the negative edge-energy off-set $\Delta E$ is sufficiently large in absolute value to overcome the bandwidth of the lower continuum. Indeed, at second order in $U / V$ and $J_{2} / J_{1}$, one finds $\mathcal{W}=2|\Delta E|\left[1-6 V / U+10(V / U)^{2}\right]$, such that edge modes are present for all values of $U$. However, while for $V / U \gtrsim 0.1$ the edge modes are strongly localized, for larger $U$ one observes a crossover to states with very large localization length because $|\Delta E| \rightarrow \mathcal{W} / 2$.

In dimerization $D 2$, both for $U \ll J_{1} \ll V$ and $J_{1} \ll V \ll U$, one obtains $\Delta E=-J_{1}^{2} / V$, such that in the limit $J_{2} \ll J_{1}, \Delta E \approx-\mu / 2$. This substantial edge correction is at the origin of the strongly localized edge modes that appear in between the two continua (ii) or well below the lower continuum (iv).

These predictions exactly agree with the numerical findings in the appropriate interaction regimes. Moreover, the effective single-particle ionic Hubbard Tamm analysis distinctly highlights the role of nearest-neighbor interactions behind the existence and the localization properties of the $D 1 / d_{3}, D 1 / d_{1}, D 2 / \tilde{d}_{3}$ EBS.

While it might seem intuitive that strong interactions localize, we recall that this is in general not true. In the case of on-site interactions only, we have proven that EBS localization is not supported in a finite system in the limit $U \rightarrow \infty$ [13]. Here, we demonstrate that the presence of nearest-neighbor interactions guarantees the existence of strongly-localized EBS in both dimerizations even for large values of $U$ and $V$.

\section{Conclusions}

In this work, we have discussed the two-body problem in a Su-Schrieffer-Heeger chain with on-site and nearest-neighbor interactions, extending the results presented in Ref. [13]. We have identified optical fiber setups as the most promising experimental realization of our predictions, thanks to the possibility of implementing large nearestneighbor interactions. We have shown that two types of bound states exist: in-cell bound states analogous to the ones found in Ref. [13] in the presence of on-site interactions only, and out-of-cell bound states that exist thanks to nearest-neighbor interactions. These two types of dimers become resonant depending on the values of $U$ and $V$, yielding bound states with mixed in-cell/out-of-cell character and larger mobility.

We have then considered open boundary conditions. We have numerically found that nearest-neighbor interactions $V$ generate strongly-localized $D 1 / d_{1} \mathrm{EBS}$, which do not exist in dimerization $D 1$ at $V=0$. In dimerization $D 2$, we have studied the fate of the two-body edge-bound state $D 2 / d_{2}$ in the presence of the novel $D 2 / \tilde{d}_{3} \mathrm{EBS}$, created by nearest-neighbor interactions. When the energies of $D 2 / d_{2}$ and $D 2 / \tilde{d}_{3} \mathrm{EBS}$ are comparable, the two edge states become hybridized. We have provided a careful characterization of the energy spectrum at the crossing.

In the strongly-interacting limit, both $D 1 / d_{1}$ and $D 2 / \tilde{d}_{3}$ EBS can be interpreted as Tamm-like states of an effective single-particle ionic Hubbard model. This model 
explains why both the $D 1 / d_{1}$ EBS and the $D 2 / \tilde{d}_{3}$ EBS persist even at very large values of on-site and nearest-neighbor interactions, preserving excellent localization properties. The presence of strongly-localized EBS for large values of interactions is a highly non-trivial result of our work.

\section{Acknowledgements}

A.R. acknowledges support from the Alexander von Humboldt foundation and W. Zwerger for the kind hospitality at the TUM. This work was supportedby the EU-FET Proactive grant AQuS, Project No. 640800 and by Provincia Autonoma di Trento, partly through the project SiQuro.

\section{References}

1. D. C. Mattis. Rev. Mod. Phys., 58 (1986), 361.

2. K. Winkler, G. Thalhammer, F. Lang, R. Grimm, J. Hecker Denschlag, A. J. Daley, A. Kantian, H. P. Büchler, and P. Zoller, Nature 441, (2006) 853.

3. J. H. Denschlag and A. J. Daley, Proceedings of the international school of physics, Enrico Fermi, Course CLXIV, Ultra-Cold Fermi Gases (2006)

4. M. Valiente and D. Petrosyan, Journal of Physics B: Atomic, Molecular and Optical Physics, 42, (2009) 121001.

5. Manuel Valiente. Phys. Rev. A, 81 (2010) 042102.

6. Manuel Valiente and Klaus Mølmer. Phys. Rev. A, 84 (2011) 053628.

7. C. Menotti, F. Minganti and A. Recati Phys. Rev. A 93 (2016), 033602

8. V. Alba, K. Saha and M. Haque, J. Stat. Mech. (2013), P10018

9. A. M. Marques and R. G. Dias, arXiv:1610.04510.

10. M. Z. Hasan and C. L. Kane, Rev. Mod. Phys., 82 (2010) 3045.

11. R. B. Laughlin, Phys. Rev. Lett., 50 (1983) 1395.

12. C.-K. Chiu, J. C. Y. Teo, A. P. Schnyder, S. Ryu, Rev. Mod. Phys. 88, (2016) 035005

13. M. Di Liberto, A. Recati, I. Carusotto and C. Menotti, Phys. Rev. A 94 (2016) 062704

14. M. A. Gorlach and A. N. Poddubny, arXiv:1608.02093

15. I. Tamm. Phys. Z. Sowjetunion, 1 (1932) 733.

16. W. Shockley. Phys. Rev., 56 (1939) 317.

17. S. Longhi, Opt. Lett., 36 (2011) 3248.

18. G. Corrielli, A. Crespi, G. Della Valle, S. Longhi, and Roberto Osellame, Nature Communications, 4 (2013) 1555.

19. A. Schreiber, A. Gábris, P. P. Rohde, K. Laiho, M. Štefaňák, V. Potoček, C. Hamilton, I. Jex, and C. Silberhorn, Science, 336 (2012), 55.

20. S. Mukherjee, A. Spracklen, D. Choudhury, N. Goldman, P. Öhberg, E. Andersson, and R. R. Thomson, New Journal of Physics, 17 (2015)115002.

21. S. Mukherjee, M. Valiente, N. Goldman, A. Spracklen, E. Andersson, P. Öhberg, and R. R. Thomson, Phys. Rev. A, 94 (2016) 053853

22. S. Baier, M. J. Mark, D. Petter, K. Aikawa, L. Chomaz, Zi Cai, M. Baranov, P. Zoller, F. Ferlaino, Science 352, (2015) 201.

23. W. P. Su, J. R. Schrieffer, and A. J. Heeger, Phys. Rev. Lett. 42, (1979) 1698.

24. M. Valiente, M. Küster and A. Saenz, Europhys. Lett. 92, (2010) 10001

25. C. Cohen-Tannoudji, J. Dupont-Roc and G. G. Grynberg, Atom-Photon Interactions: Basic Processes and Applications (John Wiley \& Sons, Inc., 1998). 